\title{
Inclusión de estudiantes ciegos en el nivel secundario común de gestión estatal de la Ciudad de Buenos Aires
}

Egle Ilva Pitton

Unidad de Evaluación Integral de la Calidad y Equidad Educativa, Ministerio de Educación, GCABA, Argentina

eglepitton@gmail.com

\author{
Fabiana Demarco \\ Unidad de Evaluación Integral de la Calidad y Equidad Educativa, Ministerio de Educación, \\ GCABA, Argentina \\ fpdemarco@gmail.com
}

\section{Resumen}

En el presente artículo se presentan los análisis preliminares de un estudio de caso que propone indagar la articulación entre Educación Común y Educación Especial, las condiciones institucionales y las prácticas pedagógicas que favorecen la inclusión de estudiantes ciegos en la escuela secundaria común.

Dichos análisis son parte de un estudio que se desarrolla desde el área de Investigación de la Unidad de Evaluación Integral de la Calidad y Equidad Educativa, Ministerio de Educación, GCBA. El mismo tiene como propósito comprender en qué sentidos las normativas y políticas que adhieren al actual paradigma de inclusión escolar inciden en las prácticas cotidianas de las instituciones educativas, de sus docentes y en la vida de niños y adolescentes con discapacidad que hoy asisten a instituciones estatales comunes de la Ciudad de Buenos Aires.

En esta comunicación se despliega, en particular, uno de los hallazgos del estudio: la enseñanza de contenidos específicos como eje estructurante en la construcción de la configuración de apoyo en la que participan la maestra integradora y los docentes de la escuela secundaria "Común".

Palabras clave: Inclusión educativa, estudiantes ciegos, escuela secundaria común, Educación Especial, configuración de apoyo 


\title{
Inclusion of blind students in the common secondary level of state of the City of Buenos Aires
}

\begin{abstract}
This article presents the preliminary analysis of a case study which suggests to investigate the relationship between Common Education and Special Education in relation to institutional conditions and pedagogical practices that favour the inclusion of blind students in the common school.

These analyzes are part of a study that is developed from the Research area of the Unit for Integral Evaluation of Educational Quality and Equity, Ministry of Education, GCBA.It aims to understand in what respects the rules and policies that adhere to the current paradigm of school inclusion affect the daily practices of educational institutions, as well as their teacher's and the lives of children and adolescents with disability attending today common State institutions of the city of Buenos Aires.
\end{abstract}

It particularly seeks to understand in which ways the teaching of specific contents could become a structuring axis to the support configuration, which involves both the integrating teacher and the "regular" teachers of a "common" secondary school.

Keywords: Inclusive education, blind students, Common school, Special education, support settings

\section{Introducción}

En Argentina, como en otros países de la región, la educación común y la educación especial convivieron durante más de cien años como subsistemas paralelos creando circuitos de escolarización diferenciados (De la Vega, 2010; Donato et al., 2014). A partir de las últimas décadas del siglo XX, las fronteras entre un subsistema y otro comenzaron a resquebrajarse como resultado de la confluencia de discursos pedagógicos, normativas y políticas educativas.

El actual paradigma de inclusión escolar promueve el derecho a la escolarización y por lo tanto la plena participación de todos los niños, niñas y adolescentes en la escuela "común"1. Este paradigma propone un sistema educativo con escuelas que renuncien a cualquier tipo o mecanismo de selección y de segregación y que, frente a los problemas asociados a estos procesos, los tomen como objeto de reflexión para evitar su reproducción.

En este proceso de resignificación de los propósitos, sentidos y alcances de la escuela común y especial, la Convención sobre los Derechos de las Personas con Discapacidad que posee en Argentina rango constitucional desde el año 2008 (Ley 26.378) marca un punto de inflexión al establecer que el Estado, en tanto reconoce a las personas con discapacidad como sujetos de derecho, debe garantizarles la igualdad de oportunidades entre las que se encuentra la de educarse en escuelas comunes.

\footnotetext{
1 Desde la sanción de la Ley de Educación Nacional 26.206/2006, la educación especial es una modalidad de las ocho que conforman la educación común. Sin embargo, en el sistema educativo se mantiene el uso de la denominación "común" para designar a las escuelas graduadas. Por otra parte, bajo el actual paradigma de la inclusión plena, las denominaciones "común" y "especial" aplicadas a las unidades de un sistema escolar perderían sentido pues se entiende que las instituciones educativas, en tanto inclusivas, lo son con independencia de su modalidad o nivel y se definen como aquellas que albergan y forman a estudiantes en situaciones diversas incluyendo, entre ellas, las de discapacidad.
} 
No obstante y como afirma Sinisi, cambiar de paradigma -y de normativa- no conlleva en absoluto una transformación "de las prácticas y los procesos de exclusión que se documenta a diario en las instituciones educativas y en la sociedad. Cambiar estas prácticas implica, en primer lugar, poder visibilizarlas y reconocerlas como tales" (Sinisi, 2010: 13).

Conceptualmente es preciso aclarar que en este cambio de paradigma se ha reemplazado el término "integración" por el de "inclusión". Dado que ambos términos refieren a concepciones divergentes acerca del sujeto, de las instituciones y de todo el Sistema Educativo en general, es necesario precisarlos.

El vocablo integración está centrado en el sujeto y tiene su origen en la idea de normalización; con lo cual, es el estudiante quien -con la ayuda de la escuela especial- "debe" adecuarse a las normas de funcionamiento de las escuelas comunes (Parrilla Latas, 2002; de la Vega, 2010). Por el contrario, el paradigma de inclusión pone el foco en los cambios que deben producirse en las instituciones como parte de un sistema educativo responsable de generar las condiciones pedagógicas necesarias para que todos los estudiantes aprendan y avancen en sus conocimientos cualquiera sea su situación social, cognitiva, subjetiva (Blanco Guijarro, 2006; Escudero y Martínez, 2011; López Melero, 2011).

En la medida en que el concepto de integración se vincula con un modo de concebir la relación entre la educación especial y la escuela común que hoy se considera superado (Filidoro, 2011), utilizaremos en este trabajo el término inclusión para referirnos, en sentido amplio, a los procesos que el sistema escolar, las instituciones y los agentes promueven para hacer posible que cualquier niño, niña y joven (Skliar, 2009), cualquiera sea su situación, aprenda en la escuela común. En otras palabras, la elección de cada uno de estos términos -integración e inclusión- a lo largo del escrito connota marcos de referencia diferentes, con lo cual, no deben interpretarse como sinónimos.

Tomando como antecedentes las investigaciones de Dubrovsky et al. (2005), Di Pietro y Pitton (2012) y Donato et al. (2014), en relación con el trabajo entre educación común y especial que se viene desarrollando en la Ciudad de Buenos Aires para dar respuesta a los desafíos que implica la educación inclusiva, este trabajo pretende ser un aporte para hacer visibles prácticas pedagógicas inclusivas considerando las condiciones que las hacen posible, así como también los obstáculos que las limitan. Nuestro propósito específico en este artículo es compartir las reflexiones en torno al modo de articulación entre la escuela secundaria común y la modalidad de educación especial hallados en el estudio del proceso de escolarización de dos estudiantes ciegos.

\section{Metodología}

A fin de indagar y comprender las prácticas pedagógicas en relación con la inclusión educativa de estudiantes con discapacidad, el diseño de la investigación exploratoria en la que se inscribe este trabajo tuvo un abordaje cualitativo: a través de un estudio de caso, se buscó reconstruir dichas prácticas desde la perspectiva de diversos actores (supervisores, profesionales vinculados a la orientación escolar, directivos y docentes de educación común y especial, familias y estudiantes), atendiendo específicamente a los procesos de inclusión de dos estudiantes ciegos en el pasaje de la educación primaria a la escuela secundaria.

El trabajo de campo se desarrolló entre 2017 y 2018 e involucró instituciones y agentes de educación común y especial. Se seleccionaron escuelas reconocidas por su trayectoria 
de integración/inclusión educativa. Dado el interés por analizar el pasaje entre niveles, el trabajo de campo comenzó en 2017 una sección de séptimo grado donde asistían dos niños ciegos, y continuó en 2018 con las instituciones en las que estos estudiantes continuaron su trayectoria escolar.

Las estrategias de recolección de información consistieron, fundamentalmente, en entrevistas en profundidad a los diversos actores involucrados en los procesos de escolarización indagados y observación de clases. En cuanto a las observaciones, cabe precisar que se llevaron a cabo por dos investigadoras: la primera, enfocada fundamentalmente en registrar el clima de la clase y el intercambio entre docentes y estudiantes a propósito del contenido de enseñanza, mientras que la segunda se centró en las interacciones entre docente, maestra integradora y estudiantes con discapacidad, por un lado, y entre ellos y sus compañeros de clase, por otro. Se realizaron grabaciones de audio, registros escritos y se tomaron fotografías de las producciones de los alumnos durante las clases.

En 2017 se realizaron 23 entrevistas en profundidad (a directivos, docentes de distintas áreas, maestro integrador, celador, familias y estudiantes) y 6 observaciones de clases en las áreas de Prácticas del Lenguaje, Matemática, Ciencias Sociales, Educación Física, Inglés y Educación Plástica. Durante 2018 se llevaron a cabo 12 entrevistas y 3 observaciones de clases, en Lengua y Matemática. Las entrevistas se realizaron a profesores y profesoras de estas asignaturas, preceptores, tutores, equipo directivo, asesor pedagógico, docente de integración y supervisor del nivel medio.

El desarrollo del artículo se organiza en dos secciones. En la primera se exponen aspectos centrales del marco normativo que brinda la Ley de Educación Nacional y las Resoluciones 155 de 2011 y 311 de 2016 con relación a la educación inclusiva. En la segunda sección se avanza en la presentación del caso bajo estudio: la escolarización de dos estudiantes ciegos en el pasaje de la educación primaria a la secundaria, con foco en el trabajo de la maestra integradora que se desempeña en este último nivel. Por último, se exponen reflexiones finales que buscan nutrir el debate acerca de las condiciones que propician contextos educativos inclusivos.

\section{Aspectos centrales del marco normativo nacional como fundamento y horizonte para la construcción de prácticas educativas inclusivas}

En la bibliografía sobre la temática, la expresión "escuelas inclusivas" se reserva para las escuelas comunes que integran estudiantes con discapacidad. Sin embargo, con la Ley de Educación Nacional 26.206/06 es posible problematizar dicha idea, en tanto esta norma sostiene que la educación común se brinda en cualquiera de las ocho modalidades del sistema educativo, ${ }^{2}$ entre las que está incluida la Educación Especial.

A través de los diversos artículos, se infiere que el término común hace referencia a los criterios pedagógicos, contenidos, objetivos que deberán ser garantizados por el Estado a través del Ministerio de Educación y el Consejo Federal para el conjunto de los estudiantes según el nivel de escolaridad:"Definirá estructuras y contenidos curriculares comunes y núcleos de aprendizaje prioritarios en todos los niveles y años de la escolaridad obligatoria" (LEN, art. 84, inc. a).Sin embargo, en la misma ley también se 
utiliza el vocablo común para nombrar a las escuelas que integran el "subsistema común", idea que se intenta erradicar en tanto hace referencia al doble circuito que se pretende superar. En el siguiente artículo, por ejemplo, la expresión educación común sirve de referencia para caracterizar la función y el alcance de la Educación Especial:"brinda atención educativa en todas aquellas problemáticas específicas que no puedan ser abordadas por la educación común" (LEN, art. 42).

No obstante, otros artículos de la misma norma permiten retomar la idea central acerca de la potencialidad de pensar la Educación Especial como modalidad, es decir, como "opción organizativa y/o curricular de la educación común" que procura "dar respuesta a requerimientos específicos de formación y atender particularidades de carácter permanente o temporal, personales y/o contextuales, con el propósito de garantizar la igualdad en el derecho a la educación" (LEN, art. 17). Esto implica avanzar hacia una concepción de un sistema educativo en el que las instituciones de diferentes modalidades se articulen y complementen con el propósito de garantizar el ingreso, permanencia y egreso de los estudiantes con discapacidad en los distintos niveles del sistema.

Este principio es coherente con el actual paradigma de inclusión escolar en tanto que hacer efectivo el derecho a la educación a todos los estudiantes requiere garantizar la plena participación de todos los niños, niñas y adolescentes en escuelas que alberguen $\mathrm{y}$ formen a estudiantes en situaciones diversas, siendo la discapacidad-temporal o permanente- una de ellas.

Los derechos a la educación de las personas con discapacidad quedan expresados en el Capítulo VIII de la citada ley, destinado a la Educación Especial. En sus cuatro artículos se establece la responsabilidad de los estados nacional y provinciales como garantes del derecho a una educación inclusiva a las personas con discapacidad en todos los niveles y modalidades del sistema educativo. Se establece que la Educación Especial será tanto una oferta educativa para los estudiantes cuyas problemáticas así lo requieran como la modalidad que brinda el acompañamiento necesario para quienes transiten su escolaridad en escuelas comunes:

La Educación Especial es la modalidad del sistema educativo destinada a asegurar el derecho a la educación de las personas con discapacidades, temporales o permanentes, en todos los niveles y modalidades del Sistema Educativo. La Educación Especial se rige por el principio de inclusión educativa, de acuerdo con el inciso n) del artículo 11 de esta ley. La Educación Especial brinda atención educativa en todas aquellas problemáticas específicas que no puedan ser abordadas por la educación común. El Ministerio de Educación, Ciencia y Tecnología, en acuerdo con el Consejo Federal de Educación, garantizará la integración de los/as alumnos/as con discapacidades en todos los niveles y modalidades según las posibilidades de cada persona. (LEN, art. 42)

De dicho artículo se desprende que la Educación Especial es una oferta destinada a generar las condiciones pedagógicas y didácticas para dar respuesta a las necesidades de los estudiantes con discapacidad en las distintas etapas de su trayectoria escolar. En este sentido, la LEN es una herramienta para hacer posible la complementariedad entre las instituciones de Educación Común y Especial, a fin de ofrecer posibilidades para el logro de una trayectoria escolar completa para cada niño, niña y adolescente. Esto supone, de base, el recorrido por los tres niveles de educación obligatoria respetando sus particularidades y potencialidades.

Estos principios normativos debieran servir de fundamento para problematizar y superar ideas y prácticas que confluyeron en la conformación del sistema educativo nacional mediante el desarrollo de dos subsistemas paralelos: la Educación Común y la Educación Especial. La escuela moderna, al mismo tiempo que logró la universalización de la educación básica obligatoria, generó -como efecto de las concepciones y prácticas 
construidas en instituciones pensadas desde la lógica homogeneizante- la segregación de estudiantes que no se adaptaran a los requerimientos de la escuela graduada. La persistencia de dicha matriz puede actuar como barrera simbólica para la inclusión de estudiantes con discapacidad en la escuela común. López Melero (2011) explica que, a diferencia de las barreras físicas (tecnológicas, arquitectónicas), las simbólicas que se manifiestan en políticas, conceptos, actitudes y concreciones didácticas son las que resultan más complejas de remover.

En otros términos, el corrimiento conceptual, ideológico y normativo que implica pensar la Educación Especial como modalidad reside fundamentalmente en pasar de concebirla como un subsistema educativo destinado a los estudiantes con discapacidad, a entenderla como una herramienta para hacer efectivo el derecho a la educación de dichos estudiantes, es decir, de brindar las "oportunidades necesarias para desarrollar y fortalecer la formación integral de las personas a lo largo de toda la vida y promover en cada educando/a la capacidad de definir su proyecto de vida" (LEN, art. 8).

Por su parte, las Resoluciones 155/11 y 311/16 del Consejo Federal avanzan hacia la superación de la existencia de estos dos circuitos diferenciados al hacer hincapié en la unidad del sistema educativo más allá de la modalidad en la que se escolaricen los estudiantes. Así, la Resolución 155/2011 prescribe sobre la necesidad de "brindar a los/as alumnos/as con discapacidad, más allá del tipo de escuelas al que asistan, una clara pertenencia a los niveles del sistema, superando de esta forma definiciones anteriores que aludían a subsistemas segmentados". En este mismo sentido, la Resolución 311/16 establece: "Propiciar condiciones para la inclusión escolar al interior del sistema educativo argentino para el acompañamiento de las trayectorias escolares de los/as estudiantes con discapacidad" (Res. 311/16. art. 1).

Como se señala en los lineamientos generales del Anexo I de esta última resolución, una de las condiciones para lograr el acompañamiento se vincula con la necesidad de asumir una responsabilidad compartida entre niveles y modalidades:

El Sistema Educativo asegurará el apoyo necesario para el acompañamiento de las trayectorias escolares de los/as estudiantes con discapacidad en caso que lo requieran, a partir de un trabajo corresponsable entre los niveles y las modalidades. (Res.311/16, art 1)

En el apartado siguiente, describiremos y analizaremos un caso de estudio en el que se avanza hacia la articulación y trabajo conjunto entre instituciones y agentes para generar condiciones propicias con el propósito de garantizar el derecho a la educación de estudiantes ciegos en una escuela secundaria común. Pensamos que dichas condiciones suponen la construcción de configuraciones de apoyo, entendiéndolas como sistemas abiertos en los que los individuos se vinculan recíprocamente, orientándose mutuamente entre sí (Castorina, 2007) y que, en los ámbitos educativos, asumen -siguiendo a Casal $(2014,2018)$ - los siguientes rasgos específicos:

》 Involucran distintas instancias, actores y recursos.

»Implican transformaciones en las funciones, tiempos y espacios institucionales.

» Requieren de una naturaleza flexible para adecuarse a la situación contextual en la que tiene lugar la inclusión.

» Poseen un carácter artesanal para su armado que impide su estandarización.

» Necesitan de un cálculo o planificación para la puesta a prueba, redefinible en cada situación a partir de sus efectos.

Estos rasgos representan la faceta más visible de las configuraciones de apoyo; sin embargo, resultan insuficientes para comprender su funcionamiento, dado que cada configuración es producto de ciertas operaciones simbólicas. Discernir cuáles son 
las operaciones lógicas sobre las que se construyen estas configuraciones evitaría que queden reducidas a propuestas espontáneas, dependientes de voluntades personales (Kiel, 2008).

Las operaciones simbólicas posibilitan la construcción de una red. En esta red se entraman tanto los actores como otros elementos, tales como el tiempo, el espacio, las demandas, las urgencias en juego, etc. En este sentido, es posible pensar que un apoyo puede llegar a convertirse en una configuración si están disponibles las coordenadas simbólicas para su inserción, es decir, si se cuenta con un lugar simbólico -explicitado, con algún sentido esperado y vacante para ser ocupado por un sujeto con su estilo-, la discriminación de funciones para ser desempeñadas por cada agente con su particularidad, y los tiempos y espacios físicos dispuestos para los fines específicos.

Siguiendo a Kiel, en la concepción de esta red los encuentros entre los actores no quedan entre personas sino que están atravesados por la relación con el dispositivo que se haya creado, como lugar de terceridad que regula las relaciones y las intervenciones. Asimismo, si la inclusión educativa es la finalidad de este entramado, las acciones tendrán un sentido verificable por lo que se produce, lo que implica el cálculo y la lectura de los efectos.

En síntesis, la posibilidad de armar configuraciones de apoyo es resultado de un entramado particular entre políticas, normas nacionales, jurisdiccionales, culturas institucionales, trayectorias profesionales, prácticas pedagógicas que generan condiciones para que los estudiantes con discapacidad se eduquen en la escuela "común", y es dicho entramado el que busca ser descripto y analizado en las siguientes secciones del trabajo.

\section{La trayectoria escolar de Marcelo y Fabio: una escolaridad en la Educación Común y Especial}

Luego de compartir, en 2017, la misma sección de séptimo grado en una escuela primaria común y, a contraturno, asistir a la misma escuela primaria de educación especial para ciegos, Marcelo y Fabio comienzan en 2018 el nivel secundario. Las instituciones educativas involucradas en este pasaje de nivel son un liceo (institución de Educación Común, en adelante A) y una escuela para jóvenes y adultos con discapacidad visual (en adelante B). A continuación, se describen algunos rasgos destacados de ambas instituciones.

El liceo Aes una institución donde se comparte lo que podríamos denominar una cultura educativa inclusiva en sentido amplio. Para los docentes, trabajar con estudiantes con discapacidad forma parte del desafío de recibir a "cualquier estudiante". Los siguientes testimonios dan cuenta de esta característica:

Asesor pedagógico:

Partimos de la idea que acá todo el mundo tiene derecho a estudiar y que por suerte somos diferentes. Nosotros tenemos el Proyecto Escuela que uno de los objetivos es este: el tratar de desarrollar las potencialidades que cada uno de nosotros tenemos. Nadie tiene más condiciones o menos condiciones para trabajar. Hay que tratar de despertarlas en cada uno de los chicos. (Entrevista a Asesor pedagógico)

Los estudiantes con discapacidad:

... los profesores pudieron adaptarse bien, me dictan... A algunos les costó más, a otros menos, pero por suerte vamos bien. En Geografía el profesor [...]. Hizo dos mapas en relieve. Me explica de diferentes maneras si no entiendo alguna cosa. [...] La de Biología 
también, en vez de traerme fotocopias, me trajo un documento en Word, me lo pasó con un pendrive (y así lo puedo escuchar). (Entrevista a Fabio)

Yo arranqué en el 2014, tenía 14 años. Mi primera escuela secundaria fue el [nombra la institución X]. En esa escuela la experiencia no fue muy favorable para mí [...] repetí ese año. En esa escuela, no sentí mucho apoyo de los profesores ni de mis compañeros. [...] Cuando volví a hacer primer año (en la escuela A), mi maestra integradora fue J. También ella fue una de las responsables que pasara a segundo porque hizo muy buen trabajo, hablando con los profes, ayudándome también.(Entrevista a Santiago, estudiante de $4^{0}$ año)

El último testimonio pertenece a un estudiante con discapacidad visual, Santiago, que en 2018 asistía a $4^{\circ}$ año. Esta experiencia, sumada a lo que llamamos perspectiva de educación inclusiva en sentido amplio, parece explicar por qué esta institución resulta reconocida por parte de las autoridades de la escuela primaria común y la escuela especial de jóvenes como un ámbito de escolarización deseable para que Marcelo y Fabio pudieran continuar su trayectoria escolar.

Por su parte, la escuela para jóvenes y adultos B estuvo históricamente destinada a jóvenes y adultos con discapacidad visual que no habían finalizado la primaria, o estudiantes que habiéndola terminado deseaban continuar su formación. Cabe señalar que en la jurisdicción no existen instituciones de nivel medio de Educación Especial estatal.

Antes [...] en esta escuela se hacía la primaria de adultos. Donde el alumno que no había finalizado su primaria, que tiene que ver con lo visual y/o con problemáticas sociales, la finalizaba acá.

Es algo como muy nuevo lo de la secundaria común. Si bien es verdad que hace años se viene trabajando con alumnos ciegos, antes estaba más institucionalizado para los alumnos que terminaban la primaria y, como eran adultos, empezaban en la nocturna. (Entrevista a Vicedirectora y psicopedagoga de la escuela B)

La obligatoriedad de la educación secundaria produjo que se ampliara el perfil y universo de estudiantes, acentuándose el ingreso de jóvenes que egresaban de la escuela primaria común con la edad teórica esperada y comenzaban su escolaridad secundaria común. Esta nueva demanda institucional obligó a extender los apoyos a la inclusión a las escuelas secundarias en general, no solo a las destinadas a la educación de adultos con la consecuente incidencia en el perfil institucional. En particular, se acentuó la tarea de las Maestras Integradoras (en adelante MI) que debieron construir un nuevo rol a través de un proceso de transmisión entre colegas y de la revisión de las prácticas en la escuela especial.

\subsection{Los dispositivos de apoyo a la inclusión: el trabajo de la maestra inte- gradora en la escuela secundaria}

De acuerdo con las regulaciones vigentes en la Ciudad de Buenos Aires, el

Maestro/a de Apoyo a la Integración (MI) cumple funciones dentro de la escuela común colaborando y sosteniendo los proyectos de integración de algunos/as alumnos/as, en forma individual o grupal, apoyando los procesos pedagógicos consensuados entre las escuelas comunes de nivel inicial y primario, y la escuela especial o de recuperación a la cual pertenecen. ${ }^{3}$ 
Normas complementarias amplían el alcance de esta función al nivel medio. ${ }^{4} \mathrm{Y}$ aclaramos que se trata de función ya que el MI no existe como cargo en el Estatuto del Docente de la jurisdicción, sino como un rol que puede ser desempeñado por aquellos docentes que cuentan con un cargo de Maestro de Grado en Educación Especial. ${ }^{5}$

Históricamente, y de acuerdo con lo que se conoce como modelo de integración escolar (Parrilla Latas, 2002), el MI consolidó su funcionamiento usual en la escuela común como figura de apoyo al estudiante "integrado", "con necesidades educativas especiales", "con proyecto de integración" (según fue modificándose la normativa al respecto), esto es: dirigiendo la mayoría de sus intervenciones a regular el trabajo de "estos" alumnos dentro de la sala de clases. Otro modo de concebir el trabajo del MI en las instituciones de Educación Común consiste en concebir centralmente su tarea como apoyo al proceso de escolarización de algunos estudiantes. Desde esta perspectiva, la tarea del MI no puede ser delimitada al trabajo "con esos" alumnos, sino que -justamente- se construye en un entramado de relaciones con los distintos actores escolares que participan de dicho proceso. Conforme a este paradigma, los directivos, los docentes (de Educación Común y Especial) y, por supuesto, los estudiantes, pero no solo "esos" sino todos aquellos que conforman el grupo de pares -los compañeros de clase- se conciben como partícipes y co-constructores de los procesos de escolarización que buscan apoyar las trayectorias escolares en el marco de una educación compartida que no "arrasa la diversidad" con estrategias homogeneizantes, sino que busca la construcción de "lo común" a partir de lo diverso (Terigi, 2008).

Como anticipamos, tomamos el trabajo de la MI como objeto de análisis para comprender las formas que asumen los procesos de escolarización de estudiantes en situación de discapacidad.

A diferencia de lo observado en la escuela primaria donde la MI asistía durante toda la jornada cuatro veces por semana al grado al que asistían Marcelo y Fabio, facilitándoles el acceso al contenido; en el liceo, la MI asiste una vez por semana a la institución común en días y horarios que coincidan con las horas en que los distintos profesores (en este caso de $1^{\circ}$ año) pueden recibirla. La mayor parte de sus intervenciones no tienen lugar en el salón de clases sino en la sala de profesores, ya que los docentes son los colegas corresponsables en el armado de las condiciones pedagógicas para apoyar las trayectorias escolares de los dos estudiantes con ceguera.

\subsubsection{El trabajo de la MI en contexto: modalidad de trabajo en las escuelas A $y B$}

Los modos en que las instituciones y sus actores se vinculan no son ajenos a la historia que porta cada una de las escuelas, a su cultura y a la perspectiva que ha construido y sostiene en la actualidad cada equipo directivo acerca de la función que tiene la escuela y el tipo de trabajo que debe llevar adelante en materia de inclusión (en términos amplios), y de inclusión de estudiantes con discapacidad.

Marcelo y Fabio asisten a algunos espacios de apoyo no obligatorios entre los muchos que ofrece la institución B, y no todo el turno ni todos los días como lo hacían en la institución de Educación Especial a la que asistieron durante su escolaridad primaria. Asimismo, la función de la MI también se concibe de modo diferente al desempeñado durante el nivel primario: como se anticipó, ya no se trata de "acompañar" a los estudiantes en el aula, sino de generar condiciones para promover su escolarización

4 Disposición 3239/o9. Recuperado de <https://www.buenosaires.gob.ar/sites/gcaba/files/disposic3239-2009_o.pdf>. 5 Véase art. 103 del Estatuto del Docente. 
en la institución de educación secundaria común. Así lo expresa la Vicedirectora de la escuela B: "tenemos una maestra integradora que va periódicamente a la escuela. No está dentro del aula, conversa con los profesores, hace orientaciones y sugerencias". Y en cuanto a la transcripción de materiales:

... los alumnos deben traerlo a nuestra escuela. Por eso digo, es diferente primaria a secundaria. El alumno no tiene a la maestra integradora dentro del aula, ni una ni dos veces. Solo que haya algún profesor que requiera para algo específico. (Entrevista a Vicedirectora, escuela B)

Esta modalidad de trabajo incide en la posibilidad de que la escuela común asuma el desafío de la inclusión como una tarea del colectivo docente. A continuación, caracterizaremos los entramados que se construyen entre instituciones de Común y Especial a propósito de la inclusión de los estudiantes con discapacidad y las condiciones que lo hacen posible, las cuales forman parte de los hallazgos del estudio.

\subsection{Condiciones institucionales que habilitan el trabajo con otros}

El pasaje de un nivel de escolaridad a otro genera incertidumbre en todos los estudiantes. Para Marcelo y Fabio, además, se sumaba el cambio en el enfoque de la intervención de Educación Especial en tanto dejarían de tener un acompañamiento individual diario dentro del aula. Tanto los estudiantes como sus familias y docentes de la escuela primaria suponían que dicho cambio no iba a resultar propicio para hacer frente a las nuevas responsabilidades de la escuela secundaria. Sin embargo, Marcelo y Fabio están logrando aprobar las materias al tiempo que dicen estar bien en su nueva escuela. ¿Cuáles son, entonces, las condiciones favorables que están haciendo posible que estos estudiantes transiten una buena experiencia escolar?

\subsubsection{El rol del Asesor pedagógico en la articulación del trabajo entre docentes de ambas instituciones}

En la escuela secundaria donde se llevó a cabo el trabajo de campo, se plantea un trabajo institucional que establece, desde el comienzo de la tarea para pensar la inclusión -en este caso de Marcelo, Fabio y Santiago (de $4^{\circ}$ año)- una relación simétrica entre los docentes de Común y Especial. En esa simetría, el lugar del colega en relación con el conocimiento juega un papel fundamental: hay un reconocimiento y valoración mutua de los saberes que cada uno posee, al mismo tiempo que se plantea como desafío conjunto el pensar los apoyos que requerirán los estudiantes. Así lo expresa la MI:

El trabajo es con los profesores, con los tutores, con el asesor pedagógico, con el gabinete de la escuela media [...]. Es mucho trabajo con el profesor, afuera del aula. Por ahí orientarlos en cómo trabajar cierto tema. En algunas materias específicas, puntualmente geografía y matemática, que son muy visuales, en los mapas en geografía yen los gráficos en matemática. El profe tiene el conocimiento específico, el contenido, en matemática, en geografía o en cada materia. Yo les explico que no soy profesora en ninguna de esas materias. Bueno, ellos me cuentan: "mirá, yo quiero trabajar esto". Y yo, desde mi especialidad de visuales, pienso cómo podemos trabajarlo. (Entrevista a MI)

Un aspecto relevante en la conformación de la configuración de apoyo para la inclusión de los estudiantes ciegos es el rol de coordinador que asume el Asesor pedagógico articulando el trabajo de los diferentes actores: escucha a los profesores, otorga un lugar a sus demandas y "ansiedades", al mismo tiempo que habilita la entrada de la MI como la figura que los acompañará en la organización del material y en encontrar el modo en que cada docente pueda hacer accesible su materia. Esta construcción -la forma de acercarles los contenidos de las materias y de cómo evaluar-, tiene un carácter "artesanal" 
según la asignatura, el estilo del docente, el contenido a transmitir, las condiciones de la clase, etc. No es un saber exclusivo de la MI que brinda a los docentes, sino una construcción compartida y producida en cada caso, a partir del reconocimiento del "no saber" frente a cada nueva situación.

Los actores de la escuela B involucrados reconocen haber encontrado en la escuela A esta orientación, lo que hace posible un abordaje que va "más allá del caso" y la institucionalización de prácticas inclusivas. Este abordaje, por ende, no tiene solo efectos en la trayectoria escolar de estudiantes individuales, sino que crea condiciones para que los docentes y la institución asuman el desafío de la inclusión.

Asimismo, advertimos la disposición de varios docentes a dejarse enseñar por la situación que crea la presencia de estudiantes con discapacidad visual en su clase. Esta posición dócil a lo que necesitan sus alumnos (Di Ciacia, 2003) es percibida por los estudiantes, que reconocen la flexibilidad y "adaptación" de los profesores para lograr que aprendan y "la pasen mejor".

La modalidad de operar de la MI muestra flexibilidad en la forma de implementar su tarea, la cual es habilitada por la institución: va en busca de los tiempos libres de los profesores, concurre en días y horarios rotativos, arma su agenda en función de los profesores.

La construcción de estas configuraciones con identidad propia dentro del dispositivo escolar constituyen puntos de llegada que tienen como condición previa operaciones que crean lugares vacantes para ser encarnados por diferentes actores; consecuentemente permiten el armado de lazos a partir de los cuales el otro preste su consentimiento al trabajo en conjunto (sea estudiante, directivo, padre, docente o par) y la creación de acuerdos implícitos y explícitos sobre los modos que tomarán las tareas compartidas.

En esta experiencia es posible verificar el armado de una configuración de apoyo a partir de sus efectos de inclusión. Podría aventurarse a definir cada configuración como un "invento retroactivo" en el que se manifiesta la tensión entre la intencionalidad en el armado del entramado para la inclusión y su construcción constante en la práctica, invento siempre abierto a lecturas de sus efectos y a revisiones de lo que se produce.

\subsubsection{Pensar la enseñanza como condición para dar lugar a la "terceridad": un diálogo entre docentes de Educación Común, Educación Especial y estudiantes}

Para que la configuración de apoyo se construya con vistas a la inclusión -y no a la integración- es necesario que la relación no sea "de a dos" -docente especial-docente de común/docente-alumno- (Pitton et al., 2018) sino que los elementos intervinientes sean al menos tres. Entendemos que el contenido a enseñar es el elemento privilegiado para ocupar el lugar de terceridad que permite que se verifique la inclusión a partir del acceso de los estudiantes a la escena educativa y a los aprendizajes.

Asimismo, centrar la inclusión en la configuración de apoyo requiere abandonar la idea clásica de entender la enseñanza como tarea individual para pasar a concebirla como trabajo colectivo.

En esta tarea se ponen en juego instituciones de modalidades diferentes, también participan actores con formaciones, trayectorias y condiciones laborales distintas. En la escuela A, la diversidad de experiencias, roles y funciones de la MI y los profesores de cada una de las asignaturas no produjo distanciamientos sino que habilitó y potenció el despliegue del trabajo de cada uno de ellos. 
Para analizar esta cuestión, resulta pertinente tomar las reflexiones de Sadovsky en torno al trabajo colaborativo entre docentes e investigadores. Si bien sus análisis provienen de su experiencia como investigadora en el campo de la Didáctica de la matemática, los mismos son relevantes en tanto interpreta la potencialidad del trabajo colaborativo entre agentes provenientes de diferentes instituciones y culturas profesionales (Sadovsky et al., 2016). Una de las cuestiones para analizar es qué tienen en común unos y otros; en nuestro caso, qué comparten la MI y cada uno de los profesores de cada una de las materias de primer año. Parafraseando a la autora, diríamos que todos "batallan" con la enseñanza. Para la MI y para cada uno de los profesores de las asignaturas, la pregunta es cómo enseñar cada uno de los contenidos a quien no puede ver. Para todos, la enseñanza de cada una de las materias es "fuente de interrogantes, de incertidumbres, de desafíos" (2016:2).

Así se lo ha planteado el Asesor pedagógico a los profesores, la MI no es la responsable de enseñarles a Marcelo y Fabio, sino quien trabajará con los docentes para pensar juntos cómo enseñarles a estos estudiantes. Este cambio de tarea, "de transcribir al Braille" a "producir con otros", enriquece el problema de enseñar contenidos a estudiantes ciegos y evidencia que la cuestión del aprendizaje no se resuelve con hacer accesibles los textos sino que es necesario generar las condiciones didácticas para que los estudiantes ciegos puedan representarse aquello que se nombra en la clase y en los textos. Los siguientes fragmentos de entrevista ilustran acerca de la complejidad y riqueza de la tarea conjunta: ${ }^{6}$

Bueno, doy algún trabajo, tengo que anticiparme y dárselo a la Ml para que lo pase. Por ejemplo, empiezo a dar ejes cartesianos. Entonces Fabio tiene que ubicar puntos. Lo hablé con ella y ella le preparó un eje cartesiano en relieve. Esta bárbaro. Y me dijo: lo único, que no te va a poder marcar los puntos. Pero le propuse que le marcáramos del o al 1 para que tenga la medida. Vamos a ver cómo nos vamos a arreglar. Está interesante. Y a partir de ahí, mañana lo va a experimentar. Yo creo que ya con las medidas en relieve que él tiene, él va a poder ubicar el punto que hay que ubicar. (Entrevista a profesora de Matemática de Fabio)

Figuras 1 y 2. Eje cartesiano y gráfico en Braille y relieve

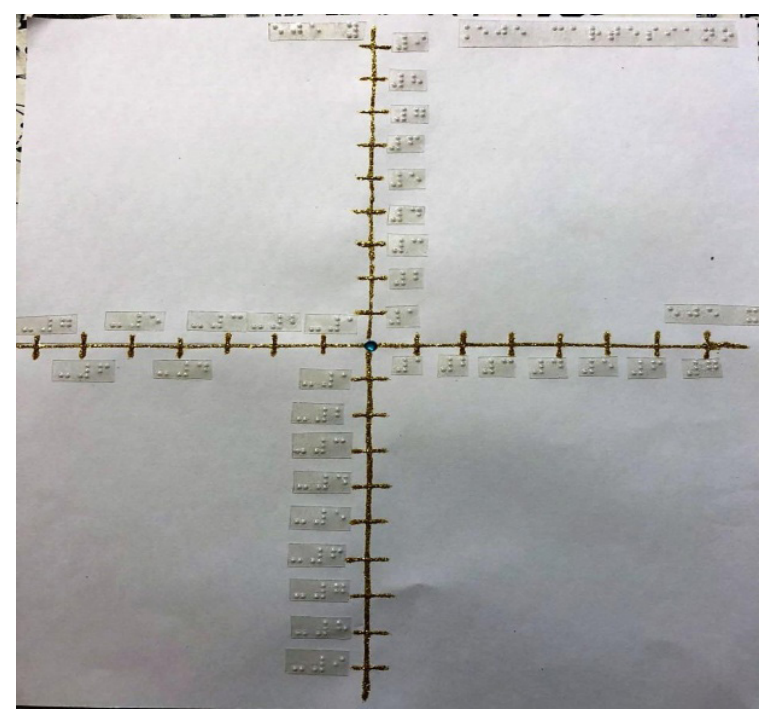




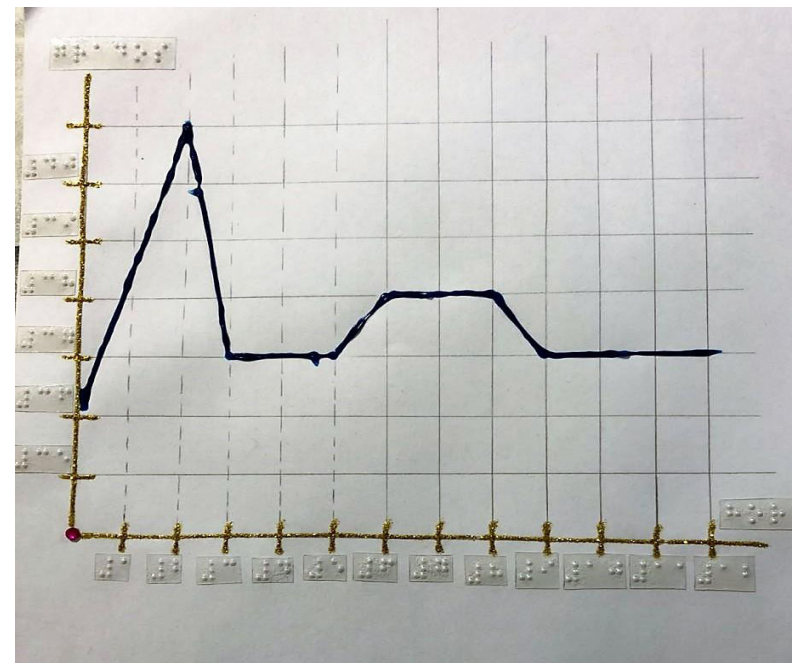

Fuente: Material elaborado por la Ml y la profesora de Matemática del liceo A

La construcción de la colaboración requiere de la

identificación de problemas comunes para los que no se dispone de respuestas ya hechas y que pueden ser explorados con los aportes de unos y otros que, desde prácticas y posicionamientos diferentes, han acumulado saberes que podrían contribuir a su tratamiento compartido. (Sadovsky et al., 2016: 9)

En este trabajo de colaboración entre la MI y los profesores, se da lugar al saber de cada uno y también al "no saber" lo que habilitará la construcción compartida de lo nuevo. La profesora de matemática de Fabio sabe el contenido pero desconocía cómo enseñárselo. La MI, a quien en un principio le parecía inviable enseñar un contenido extremadamente visual como es el de los ejes cartesianos y la representación de los puntos en un plano, "ensayó" con la profesora una posibilidad, una hipótesis de cómo podrían hacérselo accesible a Fabio.

Esta modalidad de llevar a cabo la tarea pedagógica interpela una clásica división del trabajo (Sadovsky et al., 2016), en este caso entre docentes de común y especial, pues el trabajo de la MI no se restringió a hacer accesible lo definido por la profesora de matemática sino que el trabajo conjunto llevó a construir conjuntamente cómo comunicarlo. A su vez, para la profesora de matemática implicó pensar -con la MI- qué es lo relevante para que Fabio y todos aprendan sabiendo que él no podría producir las representaciones gráficas de las funciones pero sí podría interpretarlas (Pitton et al., en prensa).

Este trabajo, además, se enmarca en una estructura que orienta y sostiene cómo es el trabajo colectivo entre los profesores de un área con el Asesor pedagógico:

Nosotros eso [el qué enseñar y evaluar] lo vemos con los coordinadores de las áreas, entonces el coordinador de exactas dialoga con los profesores de matemáticas. Porque la idea es esa: cómo podemos hacer. (Entrevista a Asesor pedagógico)

Sí, y después hay cosas que las hablo con la Ml, a ver si podemos trabajar entre las dos para explicarle tal o cual cosa, porque también es que él entienda las matemáticas y yo poder entender la forma de enseñársela a él, de llegarle con el contenido.(Entrevista a profesora de Matemática de Fabio) 
Para los docentes entrevistados, la enseñanza resulta un desafío en tanto hay un saber siempre por conquistar -tanto para el estudiante como para el profesor al pensar la forma de hacerlo accesible- al mismo tiempo que se asume que ese desafío no es en soledad.

En cuanto a las condiciones que hacen posible desplegar la potencialidad del trabajo colaborativo, Ana Espinoza (2019) expresa que la misma radica en los distintos marcos epistémicos que portan cada uno de los participantes, lo que permite ver cosas diferentes en el salón de clases. Cuando la relación entre los participantes -en este caso, profesora y MI- es simétrica, el diálogo es productivo en tanto se reconocen los distintos posicionamientos que enriquecen la mirada y la comprensión de lo que sucede en el aula.

En la pregunta sobre el qué y cómo enseñar en esta escuela, los estudiantes tienen voz para participar del diálogo en torno a las condiciones que necesitan para el aprendizaje. Así cuenta Santiago cómo encaró su primer año en el liceo, luego de haber repetido primer año en otra institución:

Lo primero que quería hacer era tratar de entenderme con los profesores y luego con los compañeros. Lo que hice fue ver con cada profesor de cada materia una forma de trabajo que se iba a hacer durante todo el año. Con todos lo pude hacer bien. Con todos tuve una forma de trabajo que me fuera fácil a mí y a la vez que ellos pudieran sentirse conformes con el modo de trabajo. (Entrevista a Santiago)

Coherente con el balance que hace Santiago, en distintas entrevistas los profesores expresan el lugar relevante que le otorgan al diálogo y al buen vínculo con los estudiantes:

Yo tengo mucho diálogo con los chicos porque ya mi materia es medio Cuco. [...] A mí me parece que es importante formar un buen vínculo viendo que hay límites entre el adolescente y el adulto. Cada uno con sus obligaciones y derechos y a partir de ahí, uno trabaja coordinando de una manera más amena porque si no, es una lucha. (Entrevista a profesora de Matemática)

Está bueno que se comprometan, que traigan propuestas y no hay que desalentar eso, me parece que la clave está ahí, en no desalentarlos y todo lo que tengan para aportar hay que escucharlos... siempre trato de mechar temas de lengua pero con cuestiones que tengan que ver con la realidad porque a los chicos les traemos tecnicismos que no tienen nada que ver con la vida de ellos. (Entrevista a profesora de Lengua)

En este sentido y desde el punto de vista de la relación pedagógica con el saber, Broitman y Charlot explican que para que el aprendizaje tenga lugar

es central la cuestión de la movilización en una actividad intelectual y, luego, la del sentido de lo que se aprende y del placer generado por esa actividad [...]. Movilizar implica poner los propios recursos en movimiento [...], lo que involucra la idea de investirse a sí mismo. (2014: 12)

Los autores expresan que, desde esta perspectiva,

es importante comprender los móviles de la movilización, las razones que llevan a un sujeto a ponerse en actividad, en movimiento, a investirse. Solo aprende quien se moviliza en una actividad intelectual: quien "estudia". ¿Por qué una persona realiza ese esfuerzo? (Broitman y Charlot, 2014: 11)

Volviendo a Santiago, haber entrado en diálogo con cada uno de sus docentes para definir cómo iba a ser el trabajo en cada materia marcó la diferencia en su forma de 
transitar su escolaridad: de negarse a estudiar a aprobar todas las materias. Ese diálogo, enmarcado por las coordenadas institucionales que incluye el trabajo con la MI, puso en "movimiento" sus propios recursos, sus propios deseos por aprender: "Sean cuales fueran las situaciones, aprender requiere siempre actividad, sentido y placer. Podría considerarse que se trata de una "ecuación pedagógica fundamental" (Broitman y Charlot, 2014: 12).

El análisis de los discursos y las prácticas en el liceo nos permite sostener que la escuela es vivida como lugar de aprendizaje no solo para los estudiantes sino también para los docentes. Si bien la frase yo esto no lo aprendí en el profesorado -tan recurrente en los discursos docentes ante situaciones complejas- está presente, la misma no se constituye en obstáculo, sino en una situación de partida que moviliza la necesidad de pensar con otros cómo generar las mejores condiciones pedagógicas para que todos aprendan. En este sentido, de todos los atravesamientos que inciden en las prácticas pedagógicas: las tradiciones escolares, la normativa y las políticas jurisdiccionales, los recursos disponibles, la articulación entre instituciones y agentes, el vínculo con las familias, entendemos que la cultura institucional juega un papel decisivo en los procesos de inclusión.

\section{A modo de cierre}

La descripción y el análisis realizado nos permiten comprender un modo de hacer escuela donde es posible reconocer ciertas condiciones que inciden fuertemente en la construcción de escuelas inclusivas.

Por un lado, las escuelas que se encuentran y entraman en este proceso cuentan con una cultura institucional y un recorrido en el cual las leyes y políticas basadas en el paradigma de los derechos sirven como fundamento de prácticas pedagógicas que ya se venían desarrollando, a la vez que potencian un posicionamiento que hace propio el desafío de constituirse en la diversidad.

Por otro lado, ambas instituciones ubican la enseñanza en el centro de los procesos de inclusión, lo que implica, en este caso, la construcción de un trabajo colectivo en el que se pone en evidencia la necesidad del encuentro entre saberes: ninguno tiene por sí solo el saber completo y absoluto sobre cómo enseñar a los estudiantes con discapacidad.

En el caso estudiado, la Educación Especial no ocupa el lugar de apoyo especializado para la inclusión sino que conforma con los docentes de la Educación Común la configuración que hace posible la participación de los estudiantes con discapacidad en las clases y en la vida cotidiana de la escuela. La tarea de la MI es más compleja y rica que lo que expresan los propios actores que la circunscriben al trabajo de transcripción y orientación a los docentes; es un trabajo de invención colectiva en el que para cada situación de enseñanza se necesita pensar y recrear las condiciones en las que será propuesta. 


\section{Q Bibliografía}

" Blanco Guijarro, R. (2006). La equidad y la inclusión social: uno de los desafíos de la educación y la escuela hoy. REICE. Revista Iberoamericana sobre Calidad, Eficacia y Cambio en Educación, 4 (3), pp. 1-15. Recuperado de <https://www.redalyc.org/ $\mathrm{pdf} / 551 / 55140302 . \mathrm{pdf}>$.

» Broitman, C. y Charlot, B. (2014). La relación con el saber. Un estudio con adultos que inician la escolaridad. Educación Matemática, 26(3), pp. 7-35. Recuperado de <http://www.memoria.fahce.unlp.edu.ar/art_revistas/pr.8373/pr.8373.pdf>.

»Casal, V. (2014). Configuraciones de apoyo para la inclusión educativa y sistemas de actividad. Avances de un estudio en el nivel inicial. Documento presentado en $\mathrm{VI}$ Congreso Internacional de Investigación y Práctica Profesional en Psicología, Buenos Aires. Recuperado de <https://www.academia.edu/7426147/CONFIGURACIONES_ DE_APOYO_para_la_inclusión_educativa_y_SISTEMAS_DE_ACTIVIDAD._ Avances_de_un_estudio_en_el_nivel_inicial >.

"Casal, V. (2018).Políticas públicas y prácticas de inclusión educativa en el nivel primario (Tesis de maestría). Facultad de Psicología, UBA, Buenos Aires. Recuperado de $<$ https://www.academia.edu/36332918/TESIS_DE_MAESTRIA>.

"Castorina, A. (2007). La "configuración" de los procesos civilizatorios, la "mentalidad histórica" y las "representaciones sociales". Algunas convergencias y diferencias. Documento presentado en XSimpósio Internacional Processo Civilizador, Campinas, Sp., Brasil. Recuperado de <http://www.uel.br/grupo-estudo/processoscivilizadores/ portugues/sitesanais/anais1o/Artigos_PDF/J_A_CASTORINA.pdf>.

»De la Vega, E. (2010). Anormales, deficientes y especiales. Genealogía de la Educación Especial. Buenos Aires: Novedades Educativas.

"Di Ciaccia, A. (2003). A proposde la pratique á plusieurs [La práctica entre varios]. Documento presentado en Rencontre PIPOL, París.

»Di Pietro S. y Pitton, E. (2012). Las configuraciones de apoyo de la Dirección de Educación Especial en escuelas primarias comunes de gestión estatal. Estudio sobre los dispositivos que brindan orientación a las escuelas para el logro de trayectorias educativas integrales y el cumplimiento de los objetivos de inclusión educativa Recuperado de <https://www. buenosaires.gob.ar/sites/gcaba/files/2012_las_configuraciones_de_apoyo_de_la_ direccion_de_educacion_especial_en_escuelas_primarias_comunes_de_gestion_ estatal_o.pdf>.

» Donato, R.; Kurlat, R.; Padín, C.; \& Rusler, V. (2014). Experiencias de inclusión educativa desde la perspectiva de aprender juntos. Estudio de casos en regiones de Argentina. Recuperado de <http://www2.congreso.gob.pe/sicr/cendocbib/con4_uibd.nsf/C347 328CE67ADD4C05257E4Boo7322Ao/\$FILE/Inclusion_Educativa.pdf>.

»Dubrovsky, S.;Navarro, A.y Rosenbaum, Z. (2005). Ilusiones y verdades acerca de la integración en la escuela común. Recuperado de<https://www.buenosaires.gob.ar/ sites/gcaba/files/2005_integracion_en_la_escuela_comun.pdf>.

»Espinoza, A. (en prensa). Una reflexión sobre el trabajo colaborativo: el diálogo como escenario para la producción de conocimiento. Buenos Aires, Argentina: UNIPE.

" Filidoro, N. (2011). La educación especial hace de lo común un momento transitorio. Ruedes, 1 (1), pp. 64-75. Recuperado de<http://bdigital.uncu.edu. $\operatorname{ar} / 3593>$. 
» Kiel, L. (2008). Un Dispositivo de Intervención en Instituciones Educativas. Recuperado de <https://www.causaclinica.com.ar/publicaciones/pub_19.html>.

»López, D. (2009). Educación especial, una modalidad del Sistema Educativo en Argentina: orientaciones I. Recuperado de <http://www.bnm.me.gov.ar/giga1/ documentos/ELooo762.pdf>.

"López Melero, M. (2011). Barreras que impiden la escuela inclusiva y algunas estrategias para construir una escuela sin exclusiones. Innovación Educativa, (21), pp. 37-54. Recuperado de<http://www.usc.es/revistas/index.php/ie/article/ view/23/140Parrilla>.

"Parrilla Latas, Á. (2002). Acerca del origen y sentido de la educación inclusiva. Revista de Educación, 327, pp. 11-29. Recuperado de <https://www.researchgate.net/profile/ Angeles_Parrillaz/publication/39155801_Acerca_del_origen_y_sentido_de_la_ educacion_inclusiva/links/oob495183fo6926c2000000o/Acerca-del-origen-ysentido-de-la-educacion-inclusiva.pdf>.

" Pitton, E.; Demarco, F. y Larripa, S. (2018). Inclusión de niños en situación de discapacidad en la escuela Primaria Común: primeros análisis a partir de un estudio exploratorio en escuelas de Gestión Estatal. Recuperado de<https://www.buenosaires. gob.ar/sites/gcaba/files/apd_inclusion_de_ninos_en_situacion_de_discapacidad_ en_la_escuela_primaria_comun.pdf>.

"Pitton, E.; Demarco, F. y Larripa, S. (2019). La Maestra de apoyo a la Integración: un análisis de dos modelos de intervención en el seguimiento de la trayectoria escolar de estudiantes ciegos. En Vinacur, T. y Bustos, J. M. (Comps). La inclusión escolar de niños, niñas y adolescentes en situación de discapacidad en la escuela común. Aportes y reflexiones del Tercer Seminario de Investigación UEICEE. pp. 59-78 Recuperado de $<$ https://oei.org.ar/wp-content/uploads/2019/12/La-inclusi\%C3\%B3n-escolar-delos-ni\%C3\%B1os.pdf

"Sadovsky, P. (2016). Tensiones y desafíos en la construcción de un trabajo colaborativo entre docentes e investigadores en didáctica de la matemática. Educación Matemática, 28 (3), pp. 9-30. Recuperado de<http://www.scielo.org.mx/scielo. php?script=sci_arttext\&pid=S1665-58262016000300009>.

» Sinisi, L. (2010). Integración o Inclusión escolar: ¿un cambio de paradigma? Boletín de Antropología y Educación (1), pp. 11-14. Recuperado de<http://antropologia. institutos.filo.uba.ar/sites/antropologia.institutos.filo.uba.ar/files/bae_no1ao2. pdf>.

"Skliar, C. (2009). Educar a cualquiera y a cada uno. Sobre el estar-juntos en la educación. Educación Especial y Educación Inclusiva. Documento presentado en VI Jornadas de Cooperación Educativa con Iberoamérica, La Antigua, Guatemala. Recuperado de <https://www.academia.edu/4253825/Educar_a_cualquiera_y_a_ cada_uno._Sobre_el_estar-juntos_en_educación>.

"Terigi, F. (2008). “Lo mismo no es lo común: La escuela común, el currículum único, el aula estándar y otros esfuerzos análogos por instituir lo común”. En Diker, G. y Frigerio, G. (Comps.), Educar: posiciones acerca de lo común, Serie Seminarios del CEM (pp. 209-221). Buenos Aires: Del Estante.

》Disposición 32/39.Dirección General de Educación de Gestión Estatal. Buenos Aires, 2009.

》 Ley N²6206. Ley de Educación Nacional, Buenos Aires, 14 de diciembre de 2006.

"Ley $\mathrm{N}^{\circ}$ 26378. Ley de aprobación de la Convención sobre los Derechos de las Personas con Discapacidad y su protocolo facultativo, resolución de la Asamblea General de las Naciones Unidas, Buenos Aires, 13 de diciembre de 2006. 
» Ordenanza $\mathrm{N}^{\circ} 40593$ (y sus modificatorias). Estatuto del Docente. Buenos Aires. Actualización abril 2019.

» Resolución CFE N ${ }^{155}$. Modalidad de Educación Especial, Buenos Aires, 13 de octubre de 2011.

» Resolución CFE N 311. Promoción, acreditación, certificación y titulación de estudiantes con discapacidad, Buenos Aires, 15 de diciembre de 2016.

\section{Egle Ilva Pitton}

Especialista en lectura, escritura y educación, Facultad Latinoamericana de Ciencias Sociales (Argentina). Licenciada en Ciencias de la Educación, Universidad de Buenos Aires.Profesora de Enseñanza Primaria, Escuela Normal Superior $\mathrm{N}^{\circ} 4$, CABA. Profesora en Institutos de formación docente y capacitadora en Grados de Aceleración. Como investigadora se desempeña en la Unidad de Evaluación Integral de la Calidad y Equidad Educativa, Ministerio de Educación, GCBA y en Proyectos UBACyT. eglepitton@gmail.com

\section{Fabiana Demarco}

Licenciada en Psicología, Universidad de Buenos Aires. Docente y coordinadora de la modalidad virtual de la Diplomatura en Inclusión Escolar con orientación en Trastornos Emocionales Severos, Universidad Nacional de Tres de Febrero. Miembro del Colectivo Entrevenir. Investigadora de la Unidad de Evaluación Integral de la Calidad y Equidad Educativa, Ministerio de Educación, GCBA. fpdemarco@gmail.com 